PROCEEDINGS OF THE

AMERICAN MATHEMATICAL SOCIETY

Volume 127, Number 2, February 1999, Pages 465-471

S 0002-9939(99)04537-2

\title{
THE WEAK CLOSURE OF THE SET OF LEFT TRANSLATION OPERATORS
}

\author{
CHING CHOU AND GUANGWU XU
}

(Communicated by J. Marshall Ash)

\begin{abstract}
It is known that for an amenable locally compact group $G, 0$ is not in the weak closure of $\{\lambda(g): g \in G\}$ of $V N(G)$. In this paper, it is proved that the converse of this is true. In other words, if $G$ is a non-amenable locally compact group, then 0 is in the weak closure of $\{\lambda(g): g \in G\}$. This answers several questions of Ülger. Applications to the algebra $C_{\delta}^{*}(G)$ and the dual of the reduced group $C^{*}$-algebra are obtained.
\end{abstract}

\section{INTRODUCTION}

Let $G$ be a locally compact group, and $A(G)$ the Fourier algebra of $G$. An element $u$ in $A(G)$ can be represented as

$$
u=f * \check{g},
$$

where $f, g \in L^{2}(G)$, and $\check{g}(s)=g\left(s^{-1}\right)$ for $s \in G$. The norm of $u$ is given by

$$
\|u\|=\inf \left\{\|f\|_{2}\|g\|_{2} ; f, g \in L^{2}(G) \text { and } u=f * \check{g}\right\} .
$$

Actually, the above infimum can be attained.

For $f \in L^{2}(G)$ and $x \in G$, the function $\lambda(x) f$ is defined by

$$
\lambda(x) f(y)=f\left(x^{-1} y\right), y \in G .
$$

So $\lambda(x)$ is a linear operator on $L^{2}(G)$ with norm 1 . Let $V N(G)$ and $C_{\delta}^{*}(G)$ be the von Neumann subalgebra and $C^{*}$-subalgebra of $\mathcal{B}\left(L^{2}(G)\right)$ generated by $\{\lambda(x): x \in$ $G$ \}, respectively. It turns out that the predual of $V N(G)$ is $A(G)$; see [3].

Let $M(G)$ denote the bounded Borel complex measures on $G$ with convolution as multiplication. Since $\mu * g \in L^{2}(G)$ for every $\mu \in M(G)$ and $g \in L^{2}(G)$, one can check that $M(G) \subseteq V N(G)$. In particular, $L^{1}(G) \subseteq V N(G)$. Let $C_{\lambda}^{*}(G)$ be the norm closure of $L^{1}(G) \subseteq V N(G)$. It is called the reduced $C^{*}$-algebra of $G$. The Banach space dual of $C_{\lambda}^{*}(G)$ is identified with a space of continuous functions on $G$, denoted by $B_{\lambda}(G)$. The Fourier-Stieltjes algebra $B(G)$ consists of continuous functions $u$ on $G$ with the form

$$
u(x)=\langle\pi(x) \xi, \eta\rangle
$$

Received by the editors January 20, 1997 and, in revised form, May 21, 1997.

1991 Mathematics Subject Classification. Primary 43A30, 46A50, 46L10; Secondary 43A07, 43A46, 46L05.

Key words and phrases. Weak closure, von Neumann algebras, Fourier algebras, amenable groups.

(C)1999 American Mathematical Society 
for some unitary representation $\pi$ of $G$ on a Hilbert space $H$ and $\xi, \eta \in H$. It is known that $A(G) \subseteq B_{\lambda}(G) \subseteq B(G)$, and both $A(G)$ and $B_{\lambda}(G)$ are ideals of $B(G)$. See $[3]$.

Note that the spaces $A(G), B_{\lambda}(G), C_{\lambda}^{*}(G)$ and $V N(G)$ have $p$-analogues for $1<$ $p<\infty$, namely, $A_{p}(G), W_{p}(G), P F_{p}(G)$ and $P M_{p}(G)$; see [2], [5], [9].

In [4], Granirer proved that for an amenable locally compact group $G$, only finite subsets of $\{\lambda(x): x \in G\}$ can be relatively weak compact in $V N(G)$. In fact, it is a consequence of the following observation: if $G$ is amenable, then $0 \notin w-\operatorname{cl}\{\lambda(x)$ : $x \in G\}$. The proof of the latter was also included in [4]. Actually, these results and some interesting applications of them (see [4], [6]) were proved in the general setting of their $p$-analogues.

On the other hand, for an infinite free set $E$ of the free group $F_{2}$ on two generators, $l^{2}\left(E^{k}\right)$ can be embedded into $V N\left(F_{2}\right), k=1,2, \ldots$; see [8], [10]. In particular, for each $k,\left\{\lambda(g) ; g \in E^{k}\right\}$ is a relatively weak compact set in $V N\left(F_{2}\right)$ and it is easy to see that $0 \in w-\operatorname{cl}\left\{\lambda(g): g \in E^{k}\right\}$. To our knowledge, there is no $p$-version of this in the literature.

The purpose of this paper is to establish a characterization of amenability based on the above discussion. More precisely, in Theorem 2.7 we show that if $G$ is a non-amenable locally compact group, then $0 \in w-\operatorname{cl}\{\lambda(x): x \in G\}$.

In a recent paper [13], Ülger made an intensive study of the weak topology of the spectrum of commutative Banach algebras. He conjectured that $G$ (which is the spectrum of $A(G)$; see [3]) is weakly closed in $V N(G)$ if and only if $G$ is amenable. He also asked what would be the situation for $P M_{p}(G)$ with $1<p<\infty$. Notice that $G$ is weakly closed in $P M_{p}(G)$ if and only if $0 \notin w-c l\{\lambda(x): x \in G\}$; see [4], [13]. So Theorem 2.7 and its $p$-version solve all the questions mentioned above.

With Theorem 2.7, we can get an improvement to the following result of Bédos [1, Theorem 1]: $G$ is amenable if and only if there exists a nonzero multiplicative linear functional on $C_{\delta}^{*}(G)$, the $C^{*}$-algebra generated by $\{\lambda(x): x \in G\}$. We are able to show in Theorem 2.9 that: $G$ is amenable if and only if there exists a linear functional $\phi$ on $C_{\delta}^{*}(G)$, such that $\inf \{|\langle\phi, \lambda(x)\rangle|: x \in G\}>0$. Note that the "if" part is the interesting part.

It is well known that a locally compact group $G$ is amenable if and only if $B(G)=B_{\lambda}(G)$. This is equivalent to saying that $1_{G} \in B_{\lambda}(G)$, where $1_{G}$ is the constant 1 function on $G$. Finally, as an application of Theorem 2.7 we prove that if $G$ is a non-amenable group, then there is a net $\left\{x_{\alpha}\right\}$ in $G$ such that

$$
u\left(x_{\alpha}\right) \rightarrow 0
$$

for every $u \in B_{\lambda}(G)$. This is formally stronger than that of $1_{G} \notin B_{\lambda}(G)$.

Every result in this paper has a $p$-version.

\section{The MAIN RESUlts}

We will begin with some discussions on the relatively weak compact sets of $\{\lambda(x): x \in G\}$.

The first result is contained in the proof of Lemma 1.2 of Granirer [4]. We give a different proof here.

Lemma 2.1. Let $G$ be a locally compact group. If there exists an infinite set $S \subseteq G$ such that $\{\lambda(x): x \in S\}$ is relatively weak compact in $V N(G)$, then $0 \in w-\operatorname{cl}\{\lambda(x)$ : $x \in G\}$. 
Proof. Choose a sequence of distinct elements $\left\{x_{n}\right\}$ of $S$ such that $\lambda\left(x_{n}\right) \rightarrow T \in$ $V N(G)$ in weak topology.

If $\left\{x_{n}\right\}$ has a subsequence (which will again be denoted by $\left\{x_{n}\right\}$ ) such that $x_{n} \rightarrow x_{0} \in G$, then $\lambda\left(x_{n}\right) \rightarrow \lambda\left(x_{0}\right)$ in $w^{*}$-topology (i.e., $u\left(x_{n}\right) \rightarrow u\left(x_{0}\right)$ for each $u \in A(G))$. So, $T=\lambda\left(x_{0}\right)$. Since $\lambda\left(x_{n}\right) \rightarrow \lambda\left(x_{0}\right)$ weakly, there is a convex combination of some $\lambda\left(x_{n_{1}}\right), \ldots, \lambda\left(x_{n_{k}}\right)$, say $\sum_{i=1}^{k} c_{i} \lambda\left(x_{n_{i}}\right)$, such that

$$
\left\|\lambda\left(x_{0}\right)-\sum_{i=1}^{k} c_{i} \lambda\left(x_{n_{i}}\right)\right\|<1
$$

Since there exists $u \in A(G)$ such that $u\left(x_{0}\right)=1, u\left(x_{n_{i}}\right)=0, i=1, \ldots, k$, and $\|u\|=1$ (so $\left\langle\lambda\left(x_{0}\right)-\sum_{i=1}^{k} c_{i} \lambda\left(x_{n_{i}}\right), u\right\rangle=1$ ), we have reached a contradiction.

So $x_{n} \rightarrow \infty$. Now for any $u \in A(G)$,

$$
\langle T, u\rangle=\lim _{n \rightarrow \infty}\left\langle\lambda\left(x_{n}\right), u\right\rangle=\lim _{n \rightarrow \infty} u\left(x_{n}\right)=0 .
$$

So we conclude that $T=0$.

Remark. We avoided the use of the facts that (i) $\operatorname{Spec} A(G)=G$ and (ii) $V N(G)$ has a topological invariant mean.

As usual, we denote by $c o E$ the convex hull of a subset $E$ of a linear space.

Lemma 2.2. If $G$ is amenable, then for any $T \in \operatorname{co}\{\lambda(x): x \in G\},\|T\|=1$.

Proof. Theorem 3.2.2 of Greenleaf [7] states that if $\mu \in M(G), \mu \geq 0$, then $\|\lambda(\mu)\|=$ $\|\mu\|$. Here if $T=\sum_{i=1}^{n} c_{i} \lambda\left(x_{i}\right)$, then $T=\lambda(\mu)$ where $\mu=\sum_{i=1}^{n} c_{i} \delta_{x_{i}}$.

The next Corollary is essentially Lemma 1.2 of Granirer [4].

Corollary 2.3. If $G$ is amenable, then

(i) $0 \notin w-c l\{\lambda(x): x \in G\}$.

(ii) Only finite subsets of $\{\lambda(x): x \in G\}$ can be relatively weak compact.

Proof. If $0 \in w-\operatorname{cl}\{\lambda(x) ; x \in G\}$, then 0 is in the norm closed convex hull of $\{\lambda(x): x \in G\}$. This contradicts Lemma 2.2. That proves part (i). Part (ii) follows from Lemma 2.1 .

One of our main results is to show that the converse of (i) of Corollary 2.3 is true. We begin with a construction of a linear functional on $V N(G)$ that is positive and away from 0 on the set $\{\lambda(x): x \in G\}$.

Proposition 2.4. If $0 \notin w-\operatorname{cl}\{\lambda(x): x \in G\}$, then there exist an $\epsilon_{0}>0$ and $\phi \in V N(G)^{*}$ with $\|\phi\| \leq 1$ such that $\langle\phi, \lambda(x)\rangle \geq \epsilon_{0}$ for every $x \in G$.

Proof. Suppose that $0 \notin w-\operatorname{cl}\{\lambda(x): x \in G\}$. Then there exist $\phi_{1}, \ldots, \phi_{k} \in$ $V N(G)^{*}$, all are of norm 1 , and an $\epsilon>0$ such that

$$
\{\lambda(x): x \in G\} \cap\left(\bigcap_{i=1}^{k}\left\{F \in V N(G):\left|\left\langle\phi_{i}, F\right\rangle\right|<\epsilon\right\}\right)=\emptyset .
$$


If for each $i$ we denote

$$
P_{i}=\left\{x \in G:\left|\left\langle\phi_{i}, \lambda(x)\right\rangle\right| \geq \epsilon\right\},
$$

then $G=\bigcup_{i=1}^{k} P_{i}$. This gives that

$$
\sum_{i=1}^{k}\left|\left\langle\phi_{i}, \lambda(x)\right\rangle\right| \geq \epsilon
$$

for every $x \in G$.

By Goldstine's theorem, we can choose a net $\left\{u_{\alpha, i}\right\}$ in $A(G)$ such that $u_{\alpha, i} \stackrel{w^{*}}{\rightarrow} \phi_{i}$ and $\left\|u_{\alpha, i}\right\| \leq 1, i=1, \ldots, k$. Assume that

$$
u_{\alpha, i}=f_{\alpha, i} * g_{\alpha, i}^{\check{\nu}}
$$

for some $f_{\alpha, i}, g_{\alpha, i} \in L^{2}(G)$ and $\left\|f_{\alpha, i}\right\|_{2}\left\|g_{\alpha, i}\right\|_{2} \leq 1$.

For each $1 \leq i \leq k$, let $v_{\alpha, i}=\left|f_{\alpha, i}\right| *\left|g_{\alpha, i}\right|$. Then $v_{\alpha, i} \in A(G)$ and $\left\|v_{\alpha, i}\right\| \leq 1$. By taking subnets if necessary, we assume that for each $i, v_{\alpha, i} \stackrel{w^{*}}{\rightarrow} \tilde{\phi}_{i}$ for some $\tilde{\phi}_{i} \in V N(G)^{*}$. Evidently, $\left\|\tilde{\phi}_{i}\right\| \leq 1$. For every $x \in G$, we have

$$
\begin{aligned}
\left|\left\langle\phi_{i}, \lambda(x)\right\rangle\right| & =\lim _{\alpha}\left|\left\langle\lambda(x), u_{\alpha, i}\right\rangle\right| \\
& =\lim _{\alpha}\left|u_{\alpha, i}(x)\right| \\
& \leq \lim _{\alpha} v_{\alpha, i}(x) \\
& =\lim _{\alpha}\left\langle\lambda(x), v_{\alpha, i}\right\rangle \\
& =\left\langle\tilde{\phi}_{i}, \lambda(x)\right\rangle,
\end{aligned}
$$

and hence

$$
\sum_{i=1}^{k}\left\langle\tilde{\phi}_{i}, \lambda(x)\right\rangle \geq \epsilon .
$$

The proposition is proved if we set $\phi=\frac{1}{k} \sum_{i=1}^{k} \tilde{\phi}_{i}$ and $\epsilon_{0}=\frac{1}{k} \epsilon$.

Lemma 2.5. If $0 \notin w-c l\{\lambda(x): x \in G\}$, then for any $T=\sum_{i=1}^{n} c_{i} \lambda\left(x_{i}\right)$ in $\operatorname{co}\{\lambda(x): x \in G\},\|T\|=1$.

Proof. Let $\phi$ and $\epsilon_{0}$ be as in Proposition 2.4. For any $T=\sum_{i=1}^{n} c_{i} \lambda\left(x_{i}\right)$ in $\operatorname{co}\{\lambda(x)$ : $x \in G\}$,

$$
\begin{aligned}
\|T\| & \geq\langle\phi, T\rangle \\
& =\sum_{i=1}^{n} c_{i}\left\langle\phi, \lambda\left(x_{i}\right)\right\rangle \geq \epsilon_{0} .
\end{aligned}
$$

Notice that $T^{k} \in \operatorname{co}\{\lambda(x): x \in G\}$ if $T \in \operatorname{co}\{\lambda(x): x \in G\}$ and $k$ is a positive integer. So,

$$
\epsilon_{0} \leq\left\|T^{k}\right\| \leq\|T\|^{k}
$$

And hence $\|T\|=1$.

The following is Proposition 9.8 of [12].

Lemma 2.6. If $\|T\|=1$ for all $T \in \operatorname{co}\{\lambda(x): x \in G\}$, then $G$ is amenable. 
Now, we are in a position to state our first main result.

Theorem 2.7. If $G$ is a non-amenable group, then $0 \in w-\operatorname{cl}\{\lambda(x): x \in G\}$ in $V N(G)$.

Proof. It follows directly from Lemma 2.5 and Lemma 2.6.

Remark. The methods described above are adoptable to the case $1<p<\infty$. Therefore, the above theorem has a $p$-analogue.

It is known that $G$ is the spectrum of the algebra $A_{p}(G)(1<p<\infty)$; see [3], [9]. Ülger conjectured that group $G$ is amenable if and only if the spectrum $G$ of $A(G)$ is weakly closed in $V N(G)$, this is the question (d)-(ii) of [13]. The other two related questions in [13] are:

(e)-(i) If $G$ is weakly closed in $V N(G)$, is $G$ weakly closed in $A_{p}(G)^{*}\left(=P M_{p}(G)\right)$ for all $p$ with $1<p<\infty$ ?

In the case where $G$ is weakly closed in $A_{p}(G)^{*}$,

(e)-(ii) Is the closedness of $G$ in $\left(A_{p}(G)^{*}\right.$, weak $)$ an intrinsic property of the algebra $A_{p}(G)$ ?

As observed in [4], [13], $G$ is weakly closed in $P M_{p}(G)$ is equivalent to saying that $0 \notin w-\operatorname{cl}\{\lambda(x): x \in G\}$ in $P M_{p}(G)$. Thus, Theorem 2.7 and its $p$-analogue answer all three questions positively. In other words, we have

Corollary 2.8. $G$ is amenable if and only if $G$ is weakly closed in $P M_{p}(G), 1<$ $p<\infty$.

A characterization of amenable locally compact groups due to Bédos is stated as: $G$ is amenable if and only if there exists a nonzero multiplicative linear functional on $C_{\delta}^{*}(G)$; see [1, Theorem 1]. Our following theorem improves Bédos' result.

Theorem 2.9. $G$ is amenable if and only if there exists a linear functional $\phi$ on $C_{\delta}^{*}(G)$ and a $\delta>0$, such that $|\langle\phi, \lambda(x)\rangle| \geq \delta$ for all $x \in G$.

Proof. If $G$ is amenable, then there exists a bounded approximate identity $\left\{u_{\alpha}\right\}$ in $A(G)$. Let $\phi$ be the restriction of a $w^{*}$-limit of $\left\{u_{\alpha}\right\}$ in $V N(G)^{*}$. Then this $\phi$ satisfies the requirement.

Conversely, suppose there exists a linear functional $\phi$ on $C_{\delta}^{*}(G)$ with the condition in the statement of the Theorem. Let $\tilde{\phi}$ be an extension of $\phi$ to $V N(G)$. If $G$ is not amenable, then by Theorem 2.7, there must be a net $\left\{x_{\alpha}\right\}$ of $G$ such that

$$
\lambda\left(x_{\alpha}\right) \rightarrow 0
$$

weakly in $V N(G)$. In particular,

$$
\left\langle\phi, \lambda\left(x_{\alpha}\right)\right\rangle=\left\langle\tilde{\phi}, \lambda\left(x_{\alpha}\right)\right\rangle \rightarrow 0 .
$$

This is a contradiction.

Let $C_{\lambda}^{*}(G)$ be the reduced group $C^{*}$-algebra of $G$ and $B_{\lambda}(G)$ its dual. It is well known that $G$ is amenable if and only if the constant 1 function is in $B_{\lambda}(G)$. Using Theorem 2.7 and a result of Granirer, we are able to obtain a formally stronger result in characterizing the amenability of locally compact groups. 
Theorem 2.10. Let $G$ be a locally compact group. Then the following are equivalent:

(i) $G$ is non-amenable.

(ii) There exists a net $\left\{x_{\alpha}\right\}$ of $G$ such that

$$
u\left(x_{\alpha}\right) \rightarrow 0
$$

for each $u \in B_{\lambda}(G)$.

Proof. By Theorem 2.7, there exists a net $\left\{x_{\alpha}\right\}$ of $G$ such that

$$
\lambda\left(x_{\alpha}\right) \rightarrow 0
$$

weakly in $V N(G)$, if $G$ is non-amenable.

Lemma 1.1 of Granirer [4] states that if $u \in B_{\lambda}(G)$, then

$$
\mu \longmapsto\langle u, \mu\rangle=\int_{G} u(x) d \mu(x)
$$

is a continuous linear functional on the normed linear space $\left(M(G),\|\cdot\|_{V N}\right)$. So,

$$
u\left(x_{\alpha}\right) \rightarrow 0
$$

as desired.

Conversely, (ii) implies that the constant 1 function does not belong to $B_{\lambda}(G)$, and hence $G$ cannot be amenable.

Remark. Both Theorem 2.9 and 2.10 have $p$-versions.

\section{ACKNOWLEDGMENT}

The main part of this work was done while the second auther was visiting the University of British Columbia. He acknowledges with thanks the support from an NSERC grant. We are indebted to Professor Edmond E. Granirer for useful conversations.

\section{REFERENCES}

1. E. Bédos, On the $C^{*}$-algebra generated by the left regular representation of a loclly compact group, Proc. Amer. Math. Soc. 120 (1994), 603-608. MR 94d:22004

2. M. Cowling, An application of Littlewood-Paley theory in harmonic analysis, Math. Ann. 241 (1979), 83-96. MR 81f:43003

3. P. Eymard, L'algèbre de Fourier d'un groupe localement compact, Bull. Soc. Math. France 92 (1964), 181-236. MR 37:4208

4. E. E. Granirer, Some results on $A_{p}(G)$ submodules of $P M_{p}(G)$, Colloq. Math. 51 (1987), 155-163. MR 88f: 43008

5. _ On some spaces of linear functionals on the algebras $A_{p}(G)$ for locally compact groups, Colloq. Math. 52 (1987), 119-132. MR 88k:43006

6. When quotients of the Fourier algebra $A(G)$ are ideals of their bidual and when $A(G)$ has WCHP, Math Japonica 46 (1997), no. 1, 69-72. CMP 97:17

7. F. P. Greenleaf, Invariant Means on Topological Groups, Van Nostrand, New York, 1969. MR 40:4776

8. U. Haagerup, An example of a nonnuclear $C^{*}$-algebra which has the metric approximation property, Invent. Math. 50 (1979), 279-293. MR 80j:46094

9. C. Herz, Harmonic synthesis for subgroups, Ann. Inst. Fourier ( Grenoble ) 23 (1973), 91-123. MR 50:7956

10. M. Leinert, Faltungsoperatoren auf gewissen diskreten gruppen, Studia Mat. 52 (1973), 149158. MR 50:7954

11. H. Leptin, On locally compact groups with invariant means, Proc. Amer. Math. Soc. 19 (1968), 489-494. MR 39:361 
12. J. Pier, Amenable Locally Compact Groups, John Wiley \& Sons, New York, 1984. MR 86a: 43001

13. A. Ülger, Some results about the spectrum of commutative Banach algebras under the weak topology and applications, Mh Math. 121 (1996), 353-379. MR 98a:46058

Department of Mathematics, State University of New York at Buffalo, Buffalo, NEW YORK 14214

E-mail address: MTHCHOU@acsu.buffalo.edu

Department of Mathematical Sciences, University of Alberta, Edmonton, Alberta, Canada T6G 1G2

E-mail address: xu@vega.math.ualberta.ca 